\title{
2080. Analytically optimal parameters of fractional-order dynamic vibration absorber
}

\author{
Yongjun Shen ${ }^{1}$, Haibo Peng ${ }^{2}$, Shaofang Wen ${ }^{3}$, Shaopu Yang ${ }^{4}$, Haijun Xing \\ ${ }_{1,4,5}$ Department of Mechanical Engineering, Shijiazhuang Tiedao University, Shijiazhuang, 050043, China \\ ${ }^{2}$ Department of Engineering Mechanics, Shijiazhuang Tiedao University, Shijiazhuang, 050043, China \\ ${ }^{3}$ Transportation Institute, Shijiazhuang Tiedao University, Shijiazhuang, 050043, China \\ ${ }^{1}$ Corresponding author \\ E-mail: ${ }^{1}$ shenyongjun@126.com, ${ }^{2} 372668319 @ q q . c o m,{ }^{3} w s f 39811 @ 163 . c o m,{ }^{4} y a n g s p @ s t d u . e d u . c n$, \\ 5xinghj@stdu.edu.cn
}

Received 9 November 2015; received in revised form 24 January 2016; accepted 17 February 2016 DOI http://dx.doi.org/10.21595/jve.2016.16617

\begin{abstract}
In this paper the optimal parameters of the fractional-order Voigt type dynamic vibration absorber (DVA) are analytically studied for two cases, named as $H_{\infty}$ and $H_{2}$ optimization criteria. At first the approximately analytical solution is obtained by the averaging method when the primary system is subjected to harmonic excitation. Then the optimal fractional coefficient and order are obtained based on $H_{\infty}$ optimization criterion, which is designed to minimize the maximum amplitude magnification factor of the primary system. Based on $\mathrm{H}_{2}$ optimization criterion, the optimal fractional parameters are obtained to reduce the total vibration energy of the primary system over the whole-frequency range. The comparisons of the approximate solutions with the numerical ones in the two cases are fulfilled, and the results verify that the approximately analytical solutions are correct and satisfactorily precise. At last the control performance of the fractional-order Voigt type DVA is compared with the classical integer-order counterpart, and it could be concluded that the fractional-order DVA has superiority in vibration engineering, and fractional-order element could replace the traditional damper and spring simultaneously in some cases.
\end{abstract}

Keywords: dynamic vibration absorber, fractional derivative, parameters optimization, averaging method.

\section{Nomenclature}

$m_{1}$

$m_{2}$

$k_{1}$

$k_{2}$

$c_{1}$

$c_{2}$

$x_{1}$

$x_{2}$

$K_{1}$

$p$

$\Gamma(z)$

$F_{0}$

$\omega$

$\omega_{1}=\sqrt{k_{1} / m_{1}}$

$\omega_{2}=\sqrt{k_{2} / m_{2}}$

$\zeta_{1}=c_{1} / 2 \omega_{1} m_{1}$

$\zeta_{2}=c_{2} / 2 \omega_{2} m_{2}$

$C_{2}(p)=c_{2}+K_{1} \omega^{p-1} \sin (p \pi / 2)$

$K_{2}(p)=k_{2}+K_{1} \omega^{p} \cos (p \pi / 2)$
Mass of the primary system

Mass of the dynamic vibration absorber

Linear stiffness coefficient of the primary system

Linear stiffness coefficient of the dynamic vibration absorber

Linear viscous damping coefficient of the primary system

Linear viscous damping coefficient of the dynamic vibration absorber

The displacement of the primary system

The displacement of the dynamic vibration absorber

The fractional coefficient

The fractional order

Gamma function

Amplitude of the excitation

Frequency of the excitation

Natural frequency of the primary system

Natural frequency of the dynamic vibration absorber

Damping ratio of the primary system

Damping ratio of the absorber

Equivalent damping coefficient

Equivalent stiffness coefficient 
$\mu=m_{2} / m_{1}$

$\lambda=\omega / \omega_{1}$

$A=\bar{a}_{1} /\left(F_{0} / k_{1}\right)$

$\bar{\zeta}_{2}=C_{2}(p) / 2 m_{2} \Omega_{2}$

$\Omega_{2}=\sqrt{K_{2}(p) / m_{2}}$

$\bar{v}=\Omega_{2} / \omega_{1}$
Mass ratio of the primary system and the absorber

Forced frequency ratio

Amplitude amplification factor

Equivalent damping ratio of the absorber

Equivalent natural frequency of the absorber

Equivalent natural frequency ratio

\section{Introduction}

The dynamic vibration absorber (DVA), also named as tuned mass damper (TMD), is a vibration control device which is attached to a vibrating primary system in order to reduce its response by appropriately designing the parameters of DVA. In 1909 Frahm [1] invented the first DVA without damping element, and it could only work in a narrow frequency range close to the natural frequency of the primary system. In 1928, Den Hartog and Ormondroyd [2] presented a DVA with damping element, and found that it could suppress the amplitude of the primary system in a broader frequency range. The DVA by Den Hartog and Ormondroyd was very typical and called as the Voigt type DVA. Because it is efficient, reliable and low-cost, the Voigt type DVA has been used in many fields of engineering practice. In order to improve the control performance, it is necessary to study the optimal parameters of the Voigt type DVA.

At present the study on Voigt type DVA can be mainly divided into the three kinds of optimization criteria, i.e. $H_{\infty}, H_{2}$, and stability maximization. The purpose of $H_{\infty}$ optimization criterion is to minimize the maximum amplitude magnification factor. This optimization criterion started from the research by Ormondroyd and Den Hartog [2], when they found that the amplitude of the primary system in the damped DVA would pass through two fixed points independent of the absorber damping. Hahnkamm [3] obtained the optimum tuning ratio in 1932, and later the optimum damping ratio was proposed by Brock in 1946 [4]. In 2002, Nishihara and Asami [5] presented the exact series solution for $H_{\infty}$ optimization criterion and compared it with the result by Den Hartog, and found that they are very close in control performance. The $\mathrm{H}_{2}$ optimization criterion was first proposed by Crandall and Mark [6] in 1963 when they considered the primary system was subjected to random excitation. The object of this criterion is to minimize the total energy of the primary system over the whole-frequency range. In other words, this object is to minimize the area under the amplitude-frequency curve of the primary system. In the late $2000 \mathrm{~s}$ Iwata [7] and Asami [8] also studied the optimum parameters respectively based on this optimization criterion. The stability maximization criterion was presented by Yamaguchi [9] in 1988 to stabilize the transient vibration of the primary system as quick as possible. All the three optimization criteria have been analytically solved for the undamped primary system. However, for the damped primary system it is difficult to obtain the analytical solution. Accordingly, numerical method was usually dopted to solve this problem. For example, Ioi and Ikeda [10], Randll [11], and Thompson [12] studied the optimum parameters and presented some important results by numerical methods. A detailed numerical study was conducted by Warburton [13] for the primary system with light damping, and he designed a table about the optimum parameters. In 1997 Nishihara and Matsuhisa [14] derived the optimum parameters for the primary system with light damping according to the stability maximization criterion. After that Asami and Nishihara $[15,16]$ presented the series solutions for the $H_{\infty}$ and $H_{2}$ optimization criteria.

Fractional-order calculus was first proposed by Hospital and Leibniz in the late 1700s. In the early development, the research was focused on the definition, properties and computation methods of the fractional-order calculus. Due to the lack of physical and mechanical meaning, it was slowly developed only as a mathematical branch [17-19]. Until Mandelbrot [20] proposed the fractal theory, the study on fractional-order calculus, especially the study on fractional-order differential equation started to develop rapidly. The research on fractional-order dynamical systems could be mainly divided into three groups, i.e. the qualitatively analysis, numerical study, 
and analytical research on the fractional-order system. The qualitatively analysis is focused on the number and stability of periodic solutions. Machado and Galhano [21], Li et al. [22], Wang and $\mathrm{Hu}$ [23], and Wang and Du [24], Rossikhin and Shitikova [25] had studied the composition of the solution for some fractional-order differential equations, and obtained some important results on its stability and properties. The numerical study is focused on the numerically investigation about the complicated nonlinear phenomenon in fractional-order dynamical system, such as bifurcation and chaos. Cao et al. [26], Sheu et al. [27] investigated the effect of the fractional-order parameters on the different nonlinear systems. The analytical research is focused on the approximate solution and the quantitative analysis of fractional-order differential equation. Wahi and Chatterjee [28], Shen and Yang [29-31], Chen and Zhu [32-35] studied the effects of fractional-order parameters on the dynamical response, and presented some important results to improve the control performance by approximately selecting fractional-order parameters.

Although the fractional-order has many advantages in engineering practice, little study on fractional-order DVA has been fulfilled. In engineering, the viscoelastic material could be modelled by fractional-order derivative, such as the air spring, metal rubber and magnetorheological elastomer. In this paper, fractional-order Voigt type DVA is introduced, and the optimum parameters of the presented DVA is studied in detail. In Section 2 the optimum fractional-order parameters, i.e. the fractional order and coefficient are obtained based on the $H_{\infty}$ and $H_{2}$ optimization criteria. The research shows that the linear damping and stiffness in the traditional Voigt type DVA can be replaced by a single fractional-order element completely in the two optimization cases. The comparisons of the approximately analytical solutions with the numerical results are fulfilled in Section 3, and the comparisons between the fractional-order and the traditional integer-order Voigt type DVA are also given in this section. Due to the advantages of fractional-order derivative, this research provides a theoretical basis to use only one fractional-order element to replace the linear spring and damper simultaneously.

\section{Analytically investigation on fractional-order DVA}

The model of fractional-order Voigt type DVA is shown in Fig. 1, which consists of the primary system $m_{1}$ and a damped DVA $m_{2}$. The fractional-order element is put between the primary system and the DVA, which is in parallel with the spring and damper of the DVA. In vibration engineering, the fractional-order element may be arbitrary viscoelastic material and its force could be modelled as:

$F_{1}=K_{1} D^{P}\left(x_{1}-x_{2}\right)$,

where $x_{1}$ and $x_{2}$ are the displacement of the primary system and the DVA, $D^{P}\left(x_{1}-x_{2}\right)$ is the $p$-order derivative of $x_{1}-x_{2}$ to $t$ with the fractional coefficient $K_{1}\left(K_{1} \geq 0\right)$ and the fractional order $p(0 \leq p \leq 1)$. There are several definitions for fractional-order derivative, and under some conditions they are equivalent. Here the Caputo's definition is adopted for simplicity:

$D^{p}[x(t)]=\frac{1}{\Gamma(1-p)} \int_{0}^{t} \frac{x^{\prime}(u)}{(t-u)^{p}} d u$,

where $\Gamma(z)$ is the Gamma function satisfying $\Gamma(z+1)=z \Gamma(z)$. In the fractional-order derivative, the initial condition is very important [36-38]. However, the steady-state responses are more meaningful in vibration engineering and both types of the derivatives will lead to the same steady-state solutions. Accordingly, the objective will be focused on the steady-state solution in the rest parts of this paper.

According to Newtonian second law, the motion equation could be established as: 
$\left\{\begin{array}{l}m_{1} \ddot{x}_{1}+c_{1} \dot{x}_{1}+k_{1} x_{1}+c_{2}\left(\dot{x}_{1}-\dot{x}_{2}\right)+k_{2}\left(x_{1}-x_{2}\right)+K_{1} D^{P}\left(x_{1}-x_{2}\right)=F_{0} \cos (\omega t), \\ m_{2} \ddot{x}_{2}-c_{2}\left(\dot{x}_{1}-\dot{x}_{2}\right)-k_{2}\left(x_{1}-x_{2}\right)-K_{1} D^{P}\left(x_{1}-x_{2}\right)=0,\end{array}\right.$

where $m_{1}, m_{2}, k_{1}, k_{2}, c_{1}, c_{2}$ are the masses, linear stiffness coefficients, and linear viscous damping coefficients of the primary system and DVA respectively. $F_{0}$ and $\omega$ are the amplitude and frequency of the external excitation.

Using the following parametric transformation:

$y_{1}=x_{1}, \quad y_{2}=x_{1}-x_{2}, \quad c_{1}=2 \zeta_{1} \omega_{1} m_{1}, \quad c_{2}=2 \zeta_{2} \omega_{2} m_{2}$,

$\omega_{1}^{2}=\frac{k_{1}}{m_{1}}, \quad \omega_{2}^{2}=\frac{k_{2}}{m_{2}}, \quad \mu=\frac{m_{2}}{m_{1}}, \quad K=\frac{K_{1}}{m_{2}}, \quad F=\frac{F_{0}}{m_{1}}$.

Eq. (2) becomes:

$\left\{\begin{array}{l}\ddot{y}_{1}+2 \zeta_{1} \omega_{1} \dot{y}_{1}+\omega_{1}^{2} y_{1}+2 \mu \zeta_{2} \omega_{2} \dot{y}_{2}+\mu \omega_{2}^{2} y_{2}+\mu K D^{p}\left(y_{2}\right)=F \cos (\omega t), \\ \ddot{y}_{1}-\ddot{y}_{2}-2 \zeta_{2} \omega_{2} \dot{y}_{2}-\omega_{2}^{2} y_{2}-K D^{p}\left(y_{2}\right)=0 .\end{array}\right.$

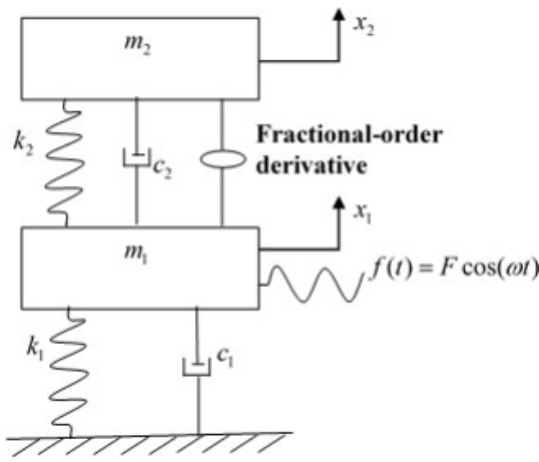

Fig. 1. The model of fractional-order DVA

\subsection{The analytical solution based on the averaging method}

According to the averaging method, one can suppose Eq. (3) has the solution as:

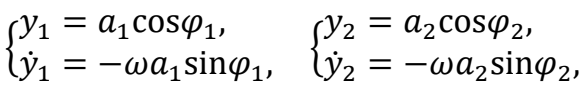

where $\varphi_{1}=\omega t+\theta_{1}, \varphi_{2}=\omega t+\theta_{2}$ are the generalized phases. By differentiating Eq. (4) to $t$ one can obtain:

$\left\{\begin{array}{l}\dot{y}_{1}=\dot{a}_{1} \cos \varphi_{1}-a_{1}\left(\omega+\dot{\theta}_{1}\right) \sin \varphi_{1}, \\ \ddot{y}_{1}=-\omega \dot{a}_{1} \sin \varphi_{1}-\omega a_{1}\left(\omega+\dot{\theta}_{1}\right) \cos \varphi_{1},\end{array}\right.$
$\left\{\begin{array}{l}\dot{y}_{2}=\dot{a}_{2} \cos \varphi_{2}-a_{2}\left(\omega+\dot{\theta}_{2}\right) \sin \varphi_{2}, \\ \ddot{y}_{2}=-\omega \dot{a}_{2} \sin \varphi_{2}-\omega a_{2}\left(\omega+\dot{\theta}_{2}\right) \cos \varphi_{2} .\end{array}\right.$

Combining Eq. (3) and Eq. (4) with Eq. (5), one can get: 
$\left\{\begin{array}{l}\dot{a}_{1} \cos \varphi_{1}-a_{1} \dot{\theta}_{1} \sin \varphi_{1}=0, \\ \dot{a}_{1} \sin \varphi_{1}+a_{1} \dot{\theta}_{1} \cos \varphi_{1}=-\frac{\Delta_{1}-\mu K D^{p}\left(a_{2} \cos \varphi_{2}\right)}{\omega},\end{array}\right.$
$\left\{\begin{array}{l}\dot{a}_{2} \cos \varphi_{2}-a_{2} \dot{\theta}_{2} \sin \varphi_{2}=0, \\ \dot{a}_{2} \sin \varphi_{2}+a_{2} \dot{\theta}_{2} \cos \varphi_{2}=-\frac{\Delta_{2}-(1+\mu) K D^{p}\left(a_{2} \cos \varphi_{2}\right)}{\omega},\end{array}\right.$

where:

$\Delta_{1}=F \cos (\omega t)+2 \zeta_{1} \omega_{1} \omega a_{1} \sin \varphi_{1}-\omega_{1}^{2} a_{1} \cos \varphi_{1}+2 \mu \zeta_{2} \omega_{2} \omega a_{2} \sin \varphi_{2}$

$-\mu \omega_{2}^{2} a_{2} \cos \varphi_{2}+a_{1} \omega^{2} \cos \varphi_{1}$,

$\Delta_{2}=F \cos (\omega t)+2 \zeta_{1} \omega_{1} \omega a_{1} \sin \varphi_{1}-\omega_{1}^{2} a_{1} \cos \varphi_{1}+2(1+\mu) \zeta_{2} \omega_{2} \omega a_{2} \sin \varphi_{2}$

$-(1+\mu) \omega_{2}^{2} a_{2} \cos \varphi_{2}+a_{2} \omega^{2} \cos \varphi_{2}$.

Solving Eq. (6) with $\dot{a}_{1}, \dot{\theta}_{1}, \dot{a}_{2}, \dot{\theta}_{2}$ as unknowns, one obtains:

$$
\begin{aligned}
& \left\{\begin{array}{l}
\dot{a}_{1}=-\frac{\Delta_{1}-\mu K D^{p}\left(a_{2} \cos \varphi_{2}\right)}{\omega} \sin \varphi_{1}, \\
a_{1} \dot{\theta}_{1}=-\frac{\Delta_{1}-\mu K D^{p}\left(a_{2} \cos \varphi_{2}\right)}{\omega} \cos \varphi_{1},
\end{array}\right. \\
& \left\{\begin{array}{l}
\dot{a}_{2}=-\frac{\Delta_{2}-(1+\mu) K D^{p}\left(a_{2} \cos \varphi_{2}\right)}{\omega} \sin \varphi_{2}, \\
a_{2} \dot{\theta}_{2}=-\frac{\Delta_{2}-(1+\mu) K D^{p}\left(a_{2} \cos \varphi_{2}\right)}{\omega} \cos \varphi_{2} .
\end{array}\right.
\end{aligned}
$$

Moreover, one could apply the standard averaging method to Eq. (7) in time interval $T$ [39-40], that means:

$$
\begin{aligned}
& \left\{\begin{array}{l}
\dot{a}_{1}=\frac{1}{T} \int_{0}^{T}\left[-\frac{\Delta_{1}-\mu K D^{p}\left(a_{2} \cos \varphi_{2}\right)}{\omega} \sin \varphi_{1}\right] d \varphi_{1}, \\
a_{1} \dot{\theta}_{1}=\frac{1}{T} \int_{0}^{T}\left[-\frac{\Delta_{1}-\mu K D^{p}\left(a_{2} \cos \varphi_{2}\right)}{\omega} \cos \varphi_{1}\right] d \varphi_{1},
\end{array}\right. \\
& \left\{\begin{array}{l}
\dot{a}_{2}=\frac{1}{T} \int_{0}^{T}\left[-\frac{\Delta_{2}-(1+\mu) K D^{p}\left(a_{2} \cos \varphi_{2}\right)}{\omega} \sin \varphi_{2}\right] d \varphi_{2}, \\
a_{2} \dot{\theta}_{2}=\frac{1}{T} \int_{0}^{T}\left[-\frac{\Delta_{2}-(1+\mu) K D^{p}\left(a_{2} \cos \varphi_{2}\right)}{\omega} \cos \varphi_{2}\right] d \varphi_{2} .
\end{array}\right.
\end{aligned}
$$

One could select the time terminal $T$ as $T=2 \pi$ if the integrands are periodic or $T=\infty$ if the integrands are aperiodic. Then Eq. (8) can be divided into the following types:

$\left\{\begin{array}{l}\dot{a}_{1}=\dot{a}_{11}+\dot{a}_{12}, \\ a_{1} \dot{\theta}_{1}=a_{1} \dot{\theta}_{11}+a_{1} \dot{\theta}_{12},\end{array} \quad\left\{\begin{array}{l}\dot{a}_{2}=\dot{a}_{21}+\dot{a}_{22}, \\ a_{2} \dot{\theta}_{2}=a_{2} \dot{\theta}_{21}+a_{2} \dot{\theta}_{22},\end{array}\right.\right.$

where:

$\left\{\begin{array}{l}\dot{a}_{11}=-\frac{1}{2 \pi} \int_{0}^{2 \pi} \frac{\Delta_{1} \sin \varphi_{1}}{\omega} d \varphi_{1}, \\ a_{1} \dot{\theta}_{11}=-\frac{1}{2 \pi} \int_{0}^{2 \pi} \frac{\Delta_{1} \cos \varphi_{1}}{\omega} d \varphi_{1},\end{array}\right.$ 


$$
\begin{aligned}
& \left\{\begin{array}{l}
\dot{a}_{12}=\lim _{T \rightarrow \infty} \frac{1}{T} \int_{0}^{T}\left[\frac{\mu K D^{p}\left(a_{2} \cos \varphi_{2}\right)}{\omega} \sin \varphi_{1}\right] d \varphi_{1}, \\
a_{1} \dot{\theta}_{12}=\lim _{T \rightarrow \infty} \frac{1}{T} \int_{0}^{T}\left[\frac{\mu K D^{p}\left(a_{2} \cos \varphi_{2}\right)}{\omega} \cos \varphi_{1}\right] d \varphi_{1},
\end{array}\right. \\
& \left\{\begin{array}{l}
\dot{a}_{21}=-\frac{1}{2 \pi} \int_{0}^{2 \pi} \frac{\Delta_{2} \sin \varphi_{2}}{\omega} d \varphi_{2}, \\
a_{2} \dot{\theta}_{21}=-\frac{1}{2 \pi} \int_{0}^{T} \frac{\Delta_{2} \cos \varphi_{2}}{\omega} d \varphi_{2},
\end{array}\right. \\
& \left\{\begin{array}{l}
\dot{a}_{22}=\lim _{T \rightarrow \infty} \frac{1}{T} \int_{0}^{2 \pi}\left[\frac{(1+\mu) K D^{p}\left(a_{2} \cos \varphi_{2}\right)}{\omega} \sin \varphi_{2}\right] d \varphi_{2}, \\
a_{2} \dot{\theta}_{22}=\lim _{T \rightarrow \infty} \frac{1}{T} \int_{0}^{T}\left[\frac{(1+\mu) K D^{p}\left(a_{2} \cos \varphi_{2}\right)}{\omega} \cos \varphi_{2}\right] d \varphi_{2} .
\end{array}\right.
\end{aligned}
$$

Then the first part of Eq. (9) will become:

$$
\begin{aligned}
& \left\{\begin{array}{c}
\dot{a}_{11}=-\frac{1}{2 \omega}\left\{F \sin \theta_{1}+2 \omega a_{1} \zeta_{1} \omega_{1}+\mu a_{2} \omega_{2}\left[2 \omega \zeta_{2} \cos \left(\theta_{1}-\theta_{2}\right)-\omega_{2} \sin \left(\theta_{1}-\theta_{2}\right)\right]\right\}, \\
a_{1} \dot{\theta}_{11}=-\frac{1}{2 \omega}\left\{F \cos \theta_{1}+a_{1}\left(\omega^{2}-\omega_{1}^{2}\right)-\mu a_{2} \omega_{2}\left[2 \omega \zeta_{2} \sin \left(\theta_{1}-\theta_{2}\right)+\omega_{2} \cos \left(\theta_{1}-\theta_{2}\right)\right]\right\},
\end{array}\right. \\
& \left\{\begin{array}{c}
\dot{a}_{21}=-\frac{1}{2 \omega}\left\{\begin{array}{c}
F \sin \theta_{2}+a_{1} \omega_{1}\left[2 \omega \zeta_{1} \cos \left(\theta_{1}-\theta_{2}\right)+\omega_{1} \sin \left(\theta_{1}-\theta_{2}\right)\right] \\
+2(1+\mu) \omega a_{2} \zeta_{2} \omega_{2}
\end{array}\right\}, \\
a_{2} \dot{\theta}_{21}=-\frac{1}{2 \omega}\left\{\begin{array}{c}
F \cos \theta_{2}+a_{1} \omega_{1}\left[2 \omega \zeta_{1} \sin \left(\theta_{1}-\theta_{2}\right)-\omega_{1} \cos \left(\theta_{1}-\theta_{2}\right)\right] \\
+a_{2}\left[\omega^{2}-(1+\mu) \omega_{2}^{2}\right]
\end{array}\right\} .
\end{array}\right.
\end{aligned}
$$

For the second part of Eq. (9), the integration region should be $[0, \infty]$. Here $a_{12}$ is taken as an example, and the others could be solved similarly. In order to calculate this integration, two important formulae are introduced [29-31]:

$$
\begin{aligned}
& \lim _{T \rightarrow \infty} \int_{0}^{T} \frac{\sin (\omega t)}{t^{p}} d t=\omega^{p-1} \Gamma(1-p) \cos \left(\frac{p \pi}{2}\right), \\
& \lim _{T \rightarrow \infty} \int_{0}^{T} \frac{\cos (\omega t)}{t^{p}} d t=\omega^{p-1} \Gamma(1-p) \sin \left(\frac{p \pi}{2}\right) .
\end{aligned}
$$

According to Eq. (12), one can get the integral in Eq. (10b):

$$
\dot{a}_{12}=\frac{\mu K a_{2} \omega^{p-1}}{2}\left[\sin \left(\theta_{1}-\theta_{2}\right) \cos \left(\frac{p \pi}{2}\right)-\cos \left(\theta_{1}-\theta_{2}\right) \sin \left(\frac{p \pi}{2}\right)\right] .
$$

The detail derivation procedures are presented in Appendix A. Similarly, one can get:

$$
\begin{aligned}
& a_{1} \dot{\theta}_{12}=\frac{\mu K a_{2} \omega^{p-1}}{2}\left[\sin \left(\theta_{1}-\theta_{2}\right) \sin \left(\frac{p \pi}{2}\right)+\cos \left(\theta_{1}-\theta_{2}\right) \cos \left(\frac{p \pi}{2}\right)\right], \\
& \dot{a}_{22}=-\frac{(1+\mu) K a_{2} \omega^{p-1}}{2} \sin \left(\frac{p \pi}{2}\right), \\
& a_{2} \dot{\theta}_{22}=\frac{(1+\mu) K a_{2} \omega^{p-1}}{2} \cos \left(\frac{p \pi}{2}\right) .
\end{aligned}
$$

Combining Eq. (13) with Eq. (11), one could obtain: 


$$
\begin{aligned}
& \int \dot{a}_{1}=-\frac{1}{2 \omega}\left\{F \sin \theta_{1}+2 \omega a_{1} \zeta_{1} \omega_{1}+\mu a_{2} \omega_{2}\left[2 \omega \zeta_{2} \cos \left(\theta_{1}-\theta_{2}\right)-\omega_{2} \sin \left(\theta_{1}-\theta_{2}\right)\right]\right\} \\
& +\frac{\mu K a_{2} \omega^{p-1}}{2}\left[\sin \left(\theta_{1}-\theta_{2}\right) \cos \left(\frac{p \pi}{2}\right)-\cos \left(\theta_{1}-\theta_{2}\right) \sin \left(\frac{p \pi}{2}\right)\right], \\
& a_{1} \dot{\theta}_{1}=-\frac{1}{2 \omega}\left\{F \cos \theta_{1}+a_{1}\left(\omega^{2}-\omega_{1}^{2}\right)-\mu a_{2} \omega_{2}\left[2 \omega \zeta_{2} \sin \left(\theta_{1}-\theta_{2}\right)+\omega_{2} \cos \left(\theta_{1}-\theta_{2}\right)\right]\right\} \\
& +\frac{\mu K a_{2} \omega^{p-1}}{2}\left[\sin \left(\theta_{1}-\theta_{2}\right) \sin \left(\frac{p \pi}{2}\right)+\cos \left(\theta_{1}-\theta_{2}\right) \cos \left(\frac{p \pi}{2}\right)\right], \\
& \dot{a}_{2}=-\frac{1}{2 \omega}\left\{F \sin \theta_{2}+a_{1} \omega_{1}\left[2 \omega \zeta_{1} \cos \left(\theta_{1}-\theta_{2}\right)+\omega_{1} \sin \left(\theta_{1}-\theta_{2}\right)\right]+2(1+\mu) \omega a_{2} \zeta_{2} \omega_{2}\right\} \\
& -\frac{(1+\mu) K a_{2} \omega^{p-1}}{2} \sin \left(\frac{p \pi}{2}\right)
\end{aligned}
$$

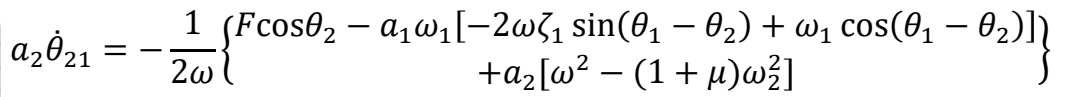

$$
\begin{aligned}
& +\frac{(1+\mu) K a_{2} \omega^{p-1}}{2} \cos \left(\frac{p \pi}{2}\right) \text {. }
\end{aligned}
$$

The steady-state system response is more meaningful. Letting the right part of Eq. (14) be zero and simplifying the equation, one can obtain:

$$
\left\{\begin{aligned}
0= & -\frac{1}{2 \omega}\left\{F \sin \bar{\theta}_{1}+2 \omega \bar{a}_{1} \zeta_{1} \omega_{1}+\mu \bar{a}_{2}\left[K \omega^{p} \sin \left(\frac{p \pi}{2}-\bar{\theta}_{1}+\bar{\theta}_{2}\right)\right.\right. \\
& \left.\left.+2 \omega \zeta_{2} \omega_{2} \cos \left(\bar{\theta}_{1}-\bar{\theta}_{2}\right)-\omega_{2}^{2} \sin \left(\bar{\theta}_{1}-\bar{\theta}_{2}\right)\right]\right\} \\
0= & \frac{1}{2 \omega}\left\{-F \cos \bar{\theta}_{1}+\bar{a}_{1}\left(-\omega^{2}+\omega_{1}^{2}\right)+\mu \bar{a}_{2}\left[K \omega^{p} \cos \left(\frac{p \pi}{2}-\bar{\theta}_{1}+\bar{\theta}_{2}\right)\right.\right. \\
& \left.\left.+2 \omega \zeta_{2} \omega_{2} \sin \left(\bar{\theta}_{1}-\bar{\theta}_{2}\right)+\omega_{2}^{2} \cos \left(\bar{\theta}_{1}-\bar{\theta}_{2}\right)\right]\right\} \\
0= & -\frac{1}{2 \omega}\left\{F \sin \bar{\theta}_{2}+\bar{a}_{1} \omega_{1}\left[2 \omega \zeta_{1} \cos \left(\bar{\theta}_{1}-\bar{\theta}_{2}\right)+\omega_{1} \sin \left(\bar{\theta}_{1}-\bar{\theta}_{2}\right)\right]\right. \\
& \left.+(1+\mu) \bar{a}_{2}\left[K \omega^{p} \sin \left(\frac{p \pi}{2}\right)+2 \omega \zeta_{2} \omega_{2}\right]\right\} \\
0= & \frac{1}{2 \omega}\left\{-F \cos \bar{\theta}_{2}+\bar{a}_{1} \omega_{1}\left[-2 \omega \zeta_{1} \sin \left(\bar{\theta}_{1}-\bar{\theta}_{2}\right)+\omega_{1} \cos \left(\bar{\theta}_{1}-\bar{\theta}_{2}\right)\right]\right. \\
& \left.+\bar{a}_{2}\left[-\omega^{2}+K(1+\mu) \omega^{p} \cos \left(\frac{p \pi}{2}\right)+(1+\mu) \omega_{2}^{2}\right]\right\} .
\end{aligned}\right.
$$

The steady-state amplitudes $\bar{a}_{1}, \bar{a}_{2}$ and phases $\bar{\theta}_{1}, \bar{\theta}_{2}$ can be obtained by solving Eq. (15). Accordingly, one can get:

$$
\bar{a}_{1}=\frac{F \sqrt{\left[K \omega^{p} \sin \left(\frac{p \pi}{2}\right)+2 \omega \zeta_{2} \omega_{2}\right]^{2}+\left[K \omega^{p} \cos \left(\frac{p \pi}{2}\right)+\omega_{2}^{2}-\omega^{2}\right]^{2}}}{\sqrt{\Delta_{3}}}, \quad \bar{a}_{2}=\frac{F \omega^{2}}{\sqrt{\Delta_{3}}},
$$

where:

$$
\begin{aligned}
\Delta_{3} & =\left\{\left[(1+\mu) \omega^{2}-\omega_{1}^{2}\right]\left[K \omega^{p} \sin \left(\frac{p \pi}{2}\right)+2 \omega \zeta_{2} \omega_{2}\right]+2 \omega \zeta_{1} \omega_{1}\left[\omega^{2}-K \omega^{p} \cos \left(\frac{p \pi}{2}\right)-\omega_{2}^{2}\right]\right\}^{2} \\
& +\left\{-2 \omega \zeta_{1} \omega_{1}\left[K \omega^{p} \sin \left(\frac{p \pi}{2}\right)+2 \omega \zeta_{2} \omega_{2}\right]+\omega_{1}^{2}\left[-\omega^{2}+K \omega^{p} \cos \left(\frac{p \pi}{2}\right)+\omega_{2}^{2}\right]\right. \\
& \left.+\omega^{2}\left[\omega^{2}-K(1+\mu) \omega^{p} \cos \left(\frac{p \pi}{2}\right)-(1+\mu) \omega_{2}^{2}\right]\right\}^{2} .
\end{aligned}
$$


Substituting the parameters with the original ones, Eq. (16) could be transformed into:

$$
\bar{a}_{1}=\frac{F \sqrt{\left[K_{1} \omega^{p} \sin \left(\frac{p \pi}{2}\right)+\omega c_{2}\right]^{2}+\left[K_{1} \omega^{P} \cos \left(\frac{p \pi}{2}\right)+k_{2}-\omega^{2} m_{2}\right]^{2}}}{\sqrt{\Delta_{4}}}, \quad \bar{a}_{2}=\frac{F \omega^{2} m_{2}}{\sqrt{\Delta_{4}}},
$$

where:

$$
\begin{aligned}
\Delta_{4} & =\left\{\omega c_{1}\left[K_{1} \omega^{p} \cos \left(\frac{p \pi}{2}\right)+k_{2}-\omega^{2} m_{2}\right]+\left[K_{1} \omega^{p} \sin \left(\frac{p \pi}{2}\right)+\omega c_{2}\right]\left[k_{1}-\omega^{2}\left(m_{1}+m_{2}\right]\right\}^{2}\right. \\
& +\left\{\omega c_{1}\left[K_{1} \omega^{p} \sin \left(\frac{p \pi}{2}\right)+\omega c_{2}\right]-k_{1}\left[K_{1} \omega^{p} \cos \left(\frac{p \pi}{2}\right)+k_{2}-\omega^{2} m_{2}\right]\right. \\
& \left.+\omega^{2} m_{2}\left[K_{1} \omega^{p} \cos \left(\frac{p \pi}{2}\right)+k_{2}\right]+\omega^{2} m_{1}\left[K_{1} \omega^{p} \cos \left(\frac{p \pi}{2}\right)+k_{2}-\omega^{2} m_{2}\right]\right\}^{2} .
\end{aligned}
$$

Two new parameters $C_{2}(p)$ and $K_{2}(p)$, defined as equivalent damping coefficient and the equivalent stiffness coefficient, can be introduced. Then Eq. (17) can be rewritten as:

$\bar{a}_{1}=\frac{F_{0} \sqrt{\omega^{2} C_{2}(p)^{2}+\left[K_{2}(p)-\omega^{2} m_{2}\right]^{2}}}{\sqrt{\Delta_{5}}}, \quad \bar{a}_{2}=\frac{F_{0} \omega^{2} m_{2}}{\sqrt{\Delta_{5}}}$,

where:

$$
\begin{aligned}
& C_{2}(p)=c_{2}+K_{1} \omega^{p-1} \sin \left(\frac{p \pi}{2}\right), \quad K_{2}(p)=k_{2}+K_{1} \omega^{p} \cos \left(\frac{p \pi}{2}\right), \\
& \Delta_{5}=\left\{\omega c_{1}\left[K_{2}(p)-\omega^{2} m_{2}\right]+\omega C_{2}(p)\left[k_{1}-\omega^{2}\left(m_{1}+m_{2}\right)\right]\right\}^{2} \\
& \quad+\left\{\omega^{2} c_{1} C_{2}(p)-k_{1}\left[K_{2}(p)-\omega^{2} m_{2}\right]+\omega^{2} m_{2} K_{2}(p)+\omega^{2} m_{1}\left[K_{2}(p)-\omega^{2} m_{2}\right]\right\}^{2} .
\end{aligned}
$$

\section{2. $H_{\infty}$ optimization of the DVA}

The calculation will be very complicated if the primary system contains viscous damping, so that the primary system without damping is considered. One could suppose $\omega \approx \omega_{1}$ in the equivalent damping coefficient and the equivalent stiffness coefficient because the main object of $H_{\infty}$ optimization criterion is to reduce the maximum resonance amplitude of the primary system. Here the amplitude amplification factor $A$ is defined based on Eq. (18) and the parameters in Eq. (3):

$$
\begin{aligned}
A & =\frac{\bar{a}_{1}}{\left(F_{0} / k_{1}\right)} \\
& =\sqrt{\frac{\left(2 \bar{v} \lambda \bar{\zeta}_{2}\right)^{2}+\left(\bar{v}^{2}-\lambda^{2}\right)^{2}}{4 \lambda^{2}\left[\zeta_{1}\left(\bar{v}^{2}-\lambda^{2}\right)+\bar{v} \bar{\zeta}_{2}\left(1-\lambda^{2}-\mu \lambda^{2}\right)\right]^{2}+\left[\bar{v}^{2}-\left(1+4 \bar{v} \zeta_{1} \bar{\zeta}_{2}+\bar{v}^{2}+\mu \bar{v}^{2}\right) \lambda^{2}+\lambda^{4}\right]^{2}}},
\end{aligned}
$$

where:

$$
\bar{\zeta}_{2}=\frac{C_{2}(p)}{2 m_{2} \Omega_{2}}, \quad \bar{v}=\frac{\Omega_{2}}{\omega_{1}}, \quad \lambda=\frac{\omega}{\omega_{1}}, \quad \Omega_{2}=\sqrt{\frac{K_{2}(p)}{m_{2}}} .
$$

Considering the undamped primary system, namely $c_{1}=0$, Eq. (19) can be: 
$\bar{A}=\frac{\bar{a}_{1}}{\left(F_{0} / k_{1}\right)}=\sqrt{\frac{\left(2 \bar{v} \lambda \bar{\zeta}_{2}\right)^{2}+\left(\bar{v}^{2}-\lambda^{2}\right)^{2}}{\left(2 \lambda \bar{v} \bar{\zeta}_{2}\right)^{2}\left(1-\lambda^{2}-\mu \lambda^{2}\right)^{2}+\left[\bar{v}^{2}-\left(1+\bar{v}^{2}+\mu \bar{v}^{2}\right) \lambda^{2}+\lambda^{4}\right]^{2}}}$.

According to the fixed-point theory, the amplitude of the primary system will pass through two fixed points independent of the damping ratio $\bar{\zeta}_{2}$. Assuming the two $\bar{A}$ to be equal when $\bar{\zeta}_{2}=0$ and $\bar{\zeta}_{2} \rightarrow \infty$, one can get:

$\bar{A}=\frac{\bar{v}^{2}-\lambda^{2}}{\bar{v}^{2}-\left(1+\bar{v}^{2}+\mu \bar{v}^{2}\right) \lambda^{2}+\lambda^{4}}=\frac{ \pm 1}{1-\lambda^{2}-\mu \lambda^{2}}$.

One can find that there is no meaning if the right part of the equal sign is positive. Taking the negative one and simplifying the equation, one could obtain:

$\lambda^{4}-\frac{2 \lambda^{2}\left(1+\bar{v}^{2}+\mu \bar{v}^{2}\right)}{2+\mu}+\frac{2 \bar{v}^{2}}{2+\mu}=0$.

Solving Eq. (22), one can get:

$\lambda_{1,2}^{2}=\frac{1+(1+\mu) \bar{v}^{2}}{2+\mu} \pm \frac{\sqrt{1-2 \bar{v}^{2}+(1+\mu)^{2} \bar{v}^{4}}}{2+\mu}$.

\subsubsection{The optimal equivalent frequency ratio}

Substituting $\lambda_{1,2}^{2}$ into $\bar{A}$ respectively when $\bar{\zeta}_{2} \rightarrow \infty$, one can obtain:

$\frac{1}{1-\lambda_{1}{ }^{2}-\mu \lambda_{1}{ }^{2}}=-\frac{1}{1-\lambda_{2}^{2}-\mu \lambda_{2}{ }^{2}}$.

Simplifying Eq. (24), one will get:

$\lambda_{1}^{2}+\lambda_{2}^{2}=\frac{2}{1+\mu}$.

According to Eq. (23), one can obtain:

$\lambda_{1}^{2}+\lambda_{2}^{2}=\frac{2\left(1+\bar{v}^{2}+\mu \bar{v}^{2}\right)}{2+\mu}$.

Comparing Eq. (25) with Eq. (26), one can get:

$\frac{2\left(1+\bar{v}^{2}+\mu \bar{v}^{2}\right)}{2+\mu}=\frac{2}{1+\mu}$.

The optimal equivalent frequency ratio can be obtained by solving Eq. (27):

$\bar{v}=\frac{1}{1+\mu}$.

Substituting $\bar{v}$ into $\lambda_{1,2}^{2}$, then the abscissas of the two fixed points can be obtained: 
$\lambda_{1,2}^{2}=\frac{1}{1+\mu}\left(1 \pm \sqrt{\frac{\mu}{2+\mu}}\right)$.

\subsubsection{The optimal equivalent damping ratio}

Substituting the optimal equivalent frequency ratio $\bar{v}$ into $\bar{A}$, differentiating $\bar{A}$ to $t$, and equating the obtained slopes to zero at the two optimal equivalent frequency ratio, one could get the best equivalent damping ratio:

$\left(\bar{\zeta}_{2}\right)_{1,2}=\sqrt{\frac{\mu}{8(1+\mu)}\left(3 \pm \sqrt{\frac{\mu}{2+\mu}}\right)}$

Based on Eq. (30), one could obtain an average value between the two given optimal equivalent damping ratios:

$\bar{\zeta}_{2}=\sqrt{\frac{\left(\bar{\zeta}_{2}\right)_{1}^{2}+\left(\bar{\zeta}_{2}\right)_{2}^{2}}{2}}=\sqrt{\frac{3 \mu}{8(1+\mu)}}$.

In order to determine the optimal equivalent stiffness and equivalent damping, the optimal frequency ratio and the optimal damping ratio are replaced by the equivalent stiffness and the equivalent damping. After simplification one could get:

$$
\left\{\begin{array}{l}
C_{2}(p)=\frac{2 m_{2} \omega_{1}}{1+\mu} \sqrt{\frac{3 \mu}{8(1+\mu)}}=c_{2}+K_{1} \omega_{1}^{p-1} \sin \left(\frac{p \pi}{2}\right), \\
K_{2}(p)=\frac{m_{2} \omega_{1}^{2}}{(1+\mu)^{2}}=k_{2}+K_{1} \omega_{1}^{p} \cos \left(\frac{p \pi}{2}\right) .
\end{array}\right.
$$

The optimal fractional order $p$ and the fractional coefficient $K_{1}$ can be obtained by solving Eq. (32):

$$
\left\{\begin{array}{l}
p=\frac{2}{\pi} \arctan \left[\frac{m_{2} \omega_{1}^{2} \sqrt{6 \mu(1+\mu)}-c_{2} \omega_{1}(1+\mu)^{2}}{2 m_{2} \omega_{1}^{2}-2 k_{2}(1+\mu)^{2}}\right] \\
K_{1}=\frac{\sqrt{\left[k_{2}-\frac{m_{2} \omega_{1}^{2}}{(1+\mu)^{2}}\right]^{2}+\left[c_{2} \omega_{1}-\frac{m_{2} \omega_{1}^{2} \sqrt{6 \mu}}{2(1+\mu)^{3 / 2}}\right]^{2}}}{\omega_{1}^{p}} .
\end{array}\right.
$$

That means, the fractional-order element could replace the linear spring and damper simultaneously in $H_{\infty}$ optimization criterion, and reduce the maximum resonance amplitude of the primary system the same as traditional Voigt type DVA.

\section{3. $\mathrm{H}_{2}$ optimization of the DVA}

$\mathrm{H}_{2}$ optimization will be more desirable if the system is subjected to random excitation instead of harmonic excitation, because the object of this optimization criterion is to reduce the total vibration energy of the primary system over the whole-frequency range. It means the area under 
the amplitude-frequency curve of the primary system should be minimized.

The performance index is defined as follow for the $\mathrm{H}_{2}$ optimization criterion:

$I_{1}=\frac{E\left[x_{1}^{2}\right]}{2 \pi S_{f} \omega_{1} / k_{1}^{2}}=\frac{\left\langle x_{1}^{2}\right\rangle}{2 \pi S_{f} \omega_{1} / k_{1}^{2}}$,

where the symbol $E[\cdot]$ is statistical average, the symbol $\langle\cdot\rangle$ is the temporal averages, and $S_{f}$ is the uniform power spectrum density of the excitation force respectively. The mean square value of the displacement of the primary system is calculated by the following equation:

$\left\langle x_{1}^{2}\right\rangle=\frac{S_{f}}{k_{1}^{2}} \int_{-\infty}^{+\infty} A^{2} d \omega=\frac{S_{f} \omega_{1}}{k_{1}^{2}} \int_{-\infty}^{+\infty} A^{2} d \lambda$,

where $A$ is the amplitude amplification factor. Thus the performance index can be simplified as:

$I_{1}=\frac{1}{2 \pi} \int_{-\infty}^{+\infty} A^{2} d \lambda$

Based on the residue theorem, Eq. (36) will be:

$I_{1}=\frac{p_{1} \bar{\zeta}_{2}^{3}+p_{2} \bar{\zeta}_{2}^{2}+p_{3} \bar{\zeta}_{2}+p_{4}}{4\left(q_{1} \bar{\zeta}_{2}^{3}+q_{2} \bar{\zeta}_{2}^{2}+q_{3} \bar{\zeta}_{2}+q_{4}\right)}$

where:

$p_{1}=4(1+\mu) \bar{v}^{2}, \quad p_{2}=4 \bar{v} \zeta_{1}\left[1+(1+\mu) \bar{v}^{2}\right]$,

$p_{3}=1+(1+\mu)^{2} \bar{v}^{4}-\bar{v}^{2}\left(2+\mu-4 \zeta_{1}^{2}\right), \quad p_{4}=\mu \bar{v}^{3} \zeta_{1}$,

$q_{1}=4 \bar{v}^{2} \zeta_{1}(1+\mu), \quad q_{2}=\bar{v}\left\{\mu+4 \zeta_{1}^{2}\left[1+\bar{v}^{2}(1+\mu)\right]\right\}$,

$q_{3}=\zeta_{1}\left[1+\bar{v}^{4}(1+\mu)^{2}-2 \bar{v}^{2}\left(1-2 \zeta_{1}^{2}\right)\right], \quad q_{4}=\mu \bar{v}^{3} \zeta_{1}^{2}$.

The performance index has a minimum value at a certain combination of $\bar{\zeta}_{2}$ and $\bar{v}$ when the value of $\mu$ and $\zeta_{1}$ is given. This condition is achieved when both the partial derivatives of $I_{1}$ with respect to $\bar{\zeta}_{2}$ and $\bar{v}$ are zero. Considering the undamped primary system, one can get a pair of simultaneous equations after differentiation:

$\left\{\begin{array}{l}\bar{\zeta}_{2}^{4}\left(4 \mu \bar{v}^{2}+4 \bar{v}^{2}\right)+\bar{\zeta}_{2}^{2}\left(3 \mu^{2} \bar{v}^{4}+6 \mu \bar{v}^{4}-\mu \bar{v}^{2}+3 \bar{v}^{4}-2 \bar{v}^{2}-1\right)=0, \\ \bar{\zeta}_{2}^{4}\left(4 \mu \bar{v}^{2}+4 \bar{v}^{2}\right)+\bar{\zeta}_{2}^{2}\left(-\mu^{2} \bar{v}^{4}-2 \mu \bar{v}^{4}+\mu \bar{v}^{2}-\bar{v}^{4}+2 \bar{v}^{2}-1\right)=0 .\end{array}\right.$

Eliminating $\bar{\zeta}_{2}$ for solving the functions, the equation about $\bar{v}$ can be obtained:

$2(1+\mu)^{2} \bar{v}^{4}-(2+\mu) \bar{v}^{2}=0$.

The optimal equivalent tuning ratio can be obtained by solving Eq. (39):

$\bar{v}_{o p t}=\frac{1}{1+\mu} \sqrt{\frac{2+\mu}{2}}$.

Substituting $\bar{v}_{\text {opt }}$ into Eq. (38), the optimal equivalent damping ratio $\bar{\zeta}_{2}$ can be obtained as: 


$$
\bar{\zeta}_{2_{o p t}}=\sqrt{\frac{\mu(4+3 \mu)}{8(1+\mu)(2+\mu)}}
$$

Replacing the parameters with the equivalent stiffness and the equivalent damping, one could get a pair of equations about $p$ and $K_{1}$ :

$$
\left\{\begin{array}{l}
C_{2}(p)=\frac{1}{2} m_{2} \omega_{1} \sqrt{\frac{\mu(4+3 \mu)}{(1+\mu)^{3}}}=c_{2}+K_{1} \omega_{1}^{p-1} \sin \left(\frac{p \pi}{2}\right), \\
K_{2}(p)=\frac{(2+\mu) m_{2} \omega_{1}^{2}}{2(1+\mu)^{2}}=k_{2}+K_{1} \omega_{1}^{p} \cos \left(\frac{p \pi}{2}\right) .
\end{array}\right.
$$

Solving the equations one can get the optimal fractional order and the fractional coefficient for $\mathrm{H}_{2}$ optimization criterion:

$$
\left\{\begin{array}{l}
p=\frac{2}{\pi} \arctan \left[\frac{2 \omega_{1} c_{2}(1+\mu)^{2}-m_{2} \omega_{1}(1+\mu) \sqrt{\mu(4+3 \mu) /(1+\mu)}}{2 k_{2}(1+\mu)^{2}-m_{2} \omega_{1}^{2}(2+\mu)}\right] \\
K_{1}=\frac{\sqrt{\left[\frac{1}{2} m_{2} \omega_{1}^{2} \sqrt{\frac{\mu(4+3 \mu)}{(1+\mu)^{3}}}-c_{2} \omega_{1}\right]^{2}+\left[\frac{(2+\mu) m_{2} \omega_{1}^{2}}{2(1+\mu)^{2}}-k_{2}\right]^{2}}}{\omega_{1}^{p}} .
\end{array}\right.
$$

That means, the fractional-order element could replace the linear spring and damper simultaneously in $\mathrm{H}_{2}$ optimization criterion, and reduce the total energy of the primary system as traditional Voigt type DVA.

\section{Numerical simulation and comparison}

\subsection{The comparison between the analytical and numerical solution}

The numerical results are also presented to verify the precision of the analytical solution. Here the numerical formula presented in $[17,18]$ are adopted:

$D^{p}\left[x\left(t_{l}\right)\right] \approx h^{-p} \sum_{j=0}^{l} C_{j}^{p} x\left(t_{l-j}\right)$,

where $t_{l}=l h$ is the sample points, $h$ is the sample step, $C_{j}^{p}$ is the binomial coefficient with the iterative relationship as:

$C_{0}^{p}=1, \quad C_{j}^{p}=\left(1-\frac{1+p}{j}\right) C_{j-1}^{p}$.

The total computation time is selected as $400 \mathrm{~s}$ and the sample step is $0.001 \mathrm{~s}$. The peak value of the later $50 \mathrm{~s}$ response is taken as the steady-state amplitude of the numerical results and the temporary response in frontal $350 \mathrm{~s}$ is omitted.

Based on the above optimized results, one could investigate the effect of the fractional-order parameters through the following four groups of parameters:

a) $k_{2}=0, c_{2}=0$. 
b) $k_{2}=0, c_{2}=100$,

c) $k_{2}=6000, c_{2}=0$

d) $k_{2}=6000, c_{2}=100$.

The other system parameters are selected as $m_{1}=1000, k_{1}=100000, c_{1}=0, m_{2}=100$, $F_{0}=1000000$.

\subsection{1. $H_{\infty}$ optimization criterion}

According to Eq. (19) and Eq. (33), the analytical normalized amplitude-frequency curves of the primary system are plotted in Fig. 2 and denoted by the solid lines. The corresponding numerical results are also shown in Fig. 2, and denoted by the circles. From the figure it could be found that the two kinds of curves agree well, that means the approximately analytical solution is satisfactorily precise. Moreover, the similarity of those curves shows that all the analytical amplitudes remain almost the same no matter how the stiffness coefficient $k_{2}$ and damping coefficient $c_{2}$ changes. That means the fractional order and fractional coefficient can affect the dynamic system characteristics by affecting the equivalent stiffness and the equivalent damping coefficient. Even it could replace the linear spring and damper of DVA completely. This result is different from the traditional results where the fractional-order derivative was generally regarded as damping device only.

\section{2. $\mathrm{H}_{2}$ optimization criterion}

Based on the aforementioned system parameters, the normalized amplitude-frequency curves are plotted in Fig. 3 according to the optimal $p$ and $K_{1}$ in Eq. (43). It could also be found that the analytical solutions agree very well with the numerical results in $H_{2}$ optimization case.

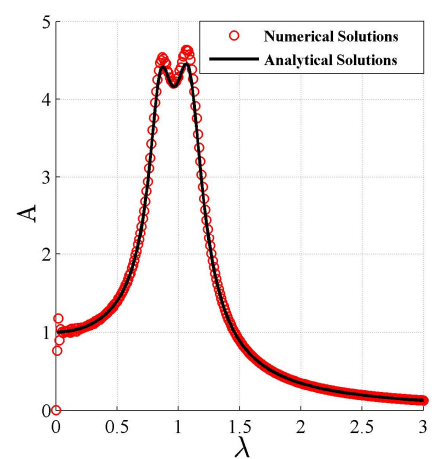

a) $k_{2}=0, c_{2}=0$

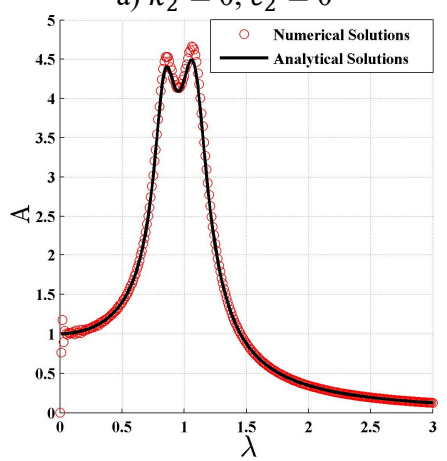

c) $k_{2}=6000, c_{2}=0$

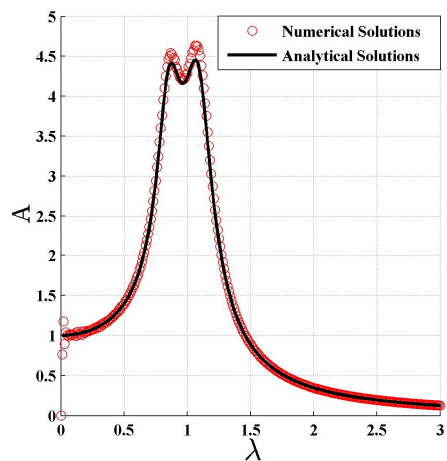

b) $k_{2}=0, c_{2}=100$

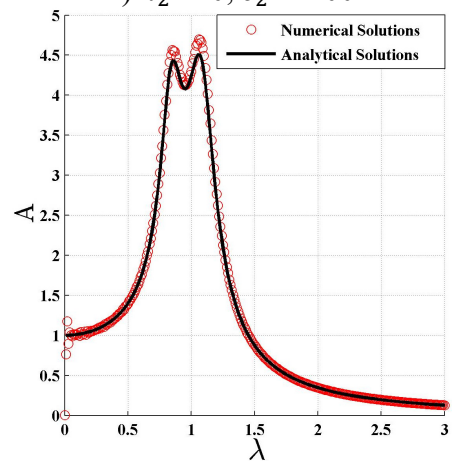

d) $k_{2}=6000, c_{2}=100$

Fig. 2. The normalized frequency response curve based on $H_{\infty}$ optimization criterion 


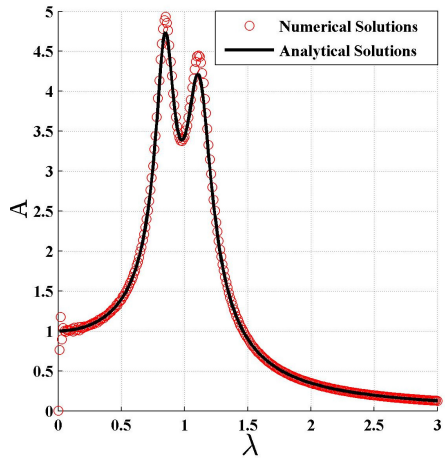

a) $k_{2}=0, c_{2}=0$

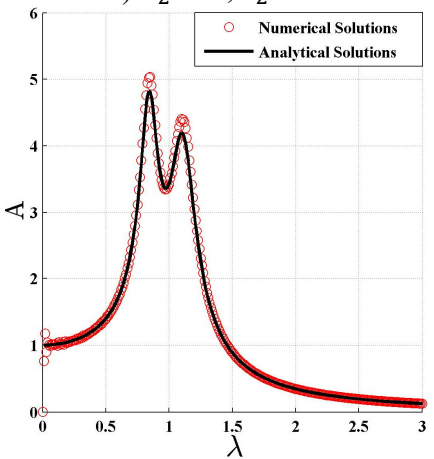

c) $k_{2}=6000, c_{2}=0$

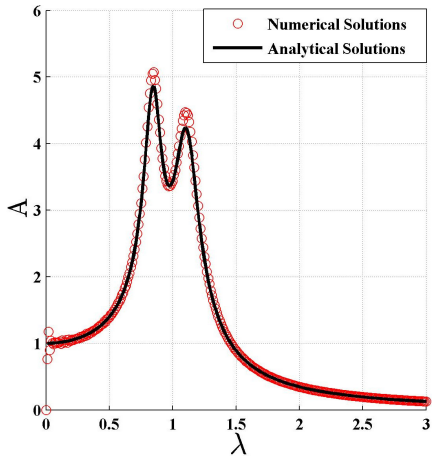

b) $k_{2}=0, c_{2}=100$

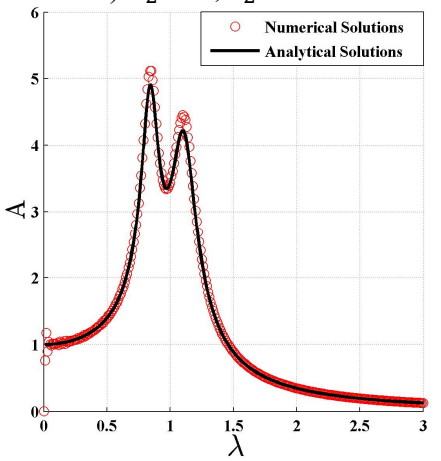

d) $k_{2}=6000, c_{2}=100$

Fig. 3. The normalized frequency response curve based on $H_{2}$ optimization criterion

To study more realistic situation, $50 \mathrm{~s}$ random excitation are constructed, which is composed of 5000 random numbers with normal distribution. The excitation is normalized so that it is with mean value 0 and variance 1 . The time history of the random excitation is shown in Fig. 4 . When there is no fractional-order derivative in the Voigt type DVA, the time history of the primary system is given in Fig. 5. Here the optimum parameters are $k_{2}=8686$ and $c_{2}=285$, which are obtained according to reference [15]. For comparison the time histories of the primary system with the fractional-order DVA for the above different system parameters are presented in Fig. 6 to Fig. 9. The variances of the primary system in the above five systems are summarized in Table 1.

Table 1. The variances of the displacement of the primary system

\begin{tabular}{|c|c|c|c|c|c|}
\hline & \multirow[b]{2}{*}{ The integer-order DVA } & \multicolumn{4}{|c|}{ The fractional-order DVA } \\
\hline & & $\begin{aligned} k_{2} & =0 \\
c_{2} & =0\end{aligned}$ & $\begin{array}{c}k_{2}=0 \\
c_{2}=100\end{array}$ & $\begin{array}{c}k_{2}=6000, \\
c_{2}=0\end{array}$ & $\begin{array}{c}k_{2}=6000 \\
c_{2}=100\end{array}$ \\
\hline Variances & $3.929 \mathrm{e}-11$ & $2.8434 \mathrm{e}-11$ & $2.8632 \mathrm{e}-11$ & $2.8348 \mathrm{e}-11$ & $2.8577 \mathrm{e}-11$ \\
\hline
\end{tabular}

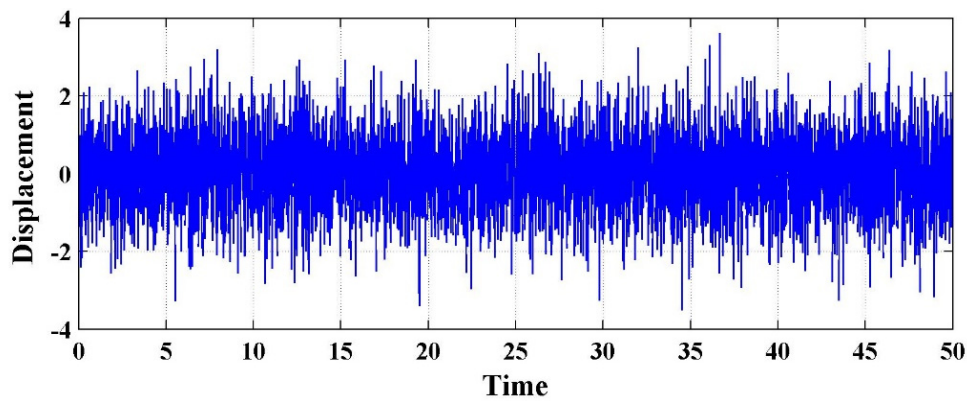

Fig. 4. The time history of random excitation 
From the Figs. 4-9 and Table 1 it could be concluded that the fractional-order DVA can not only reduce the peak displacement of the primary system $\left(H_{\infty}\right.$ optimization criterion), but also reduce the total energy of the primary system in the whole-frequency range $\left(\mathrm{H}_{2}\right.$ optimization criterion).

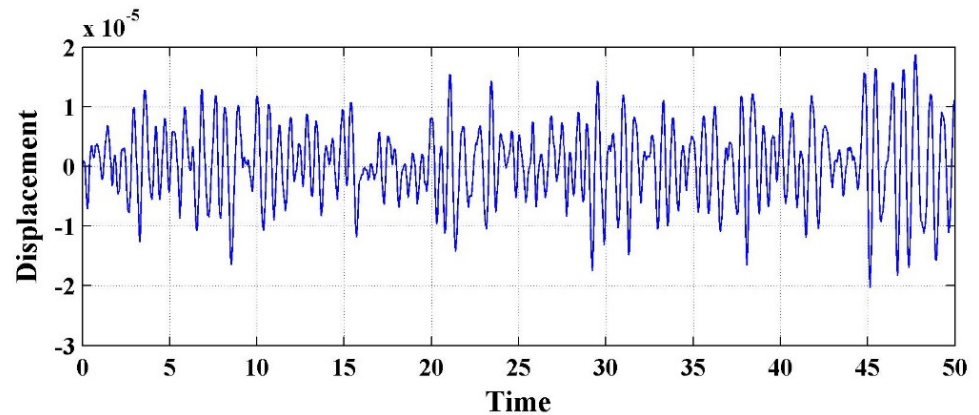

Fig. 5. The time history of the primary system without fractional-order derivative $\left(k_{2}=8686, c_{2}=285\right)$

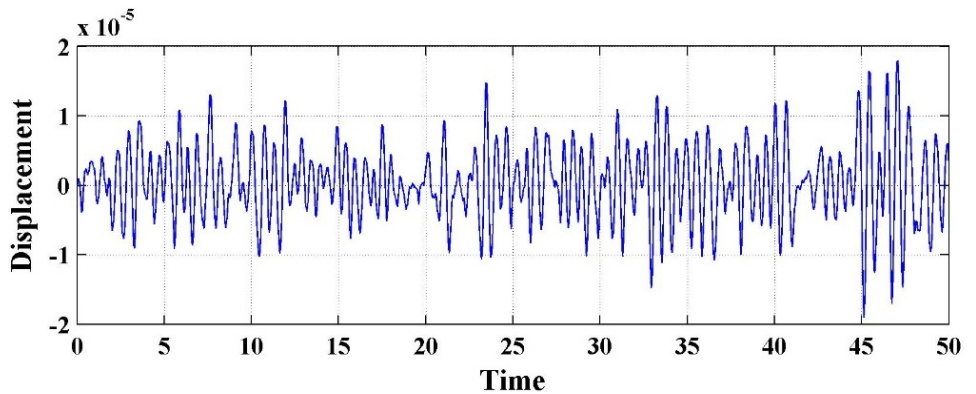

Fig. 6. The time history of the primary system with fractional-order derivative $\left(k_{2}=0, c_{2}=0\right)$

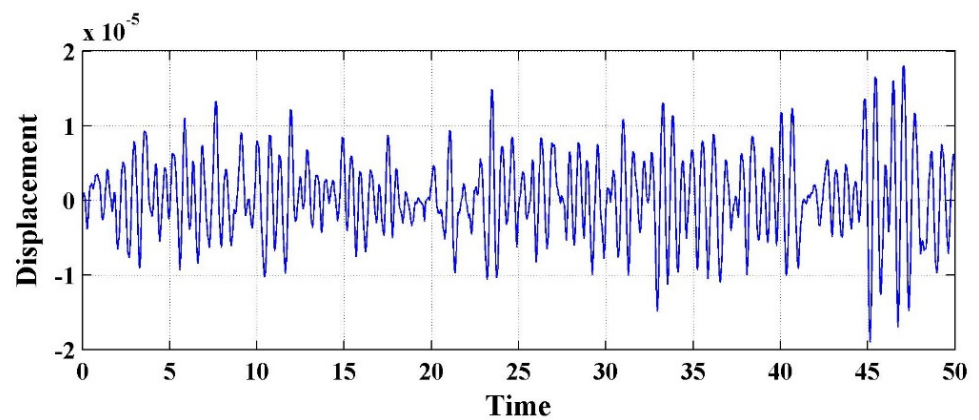

Fig. 7. The time history of the primary system with fractional-order derivative $\left(k_{2}=0, c_{2}=100\right)$

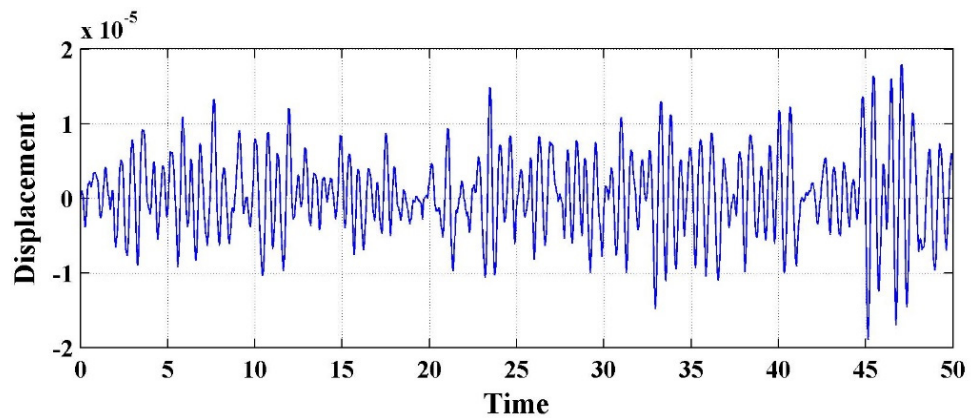

Fig. 8. The time history of the primary system with fractional-order derivative $\left(k_{2}=6000, c_{2}=0\right)$ 


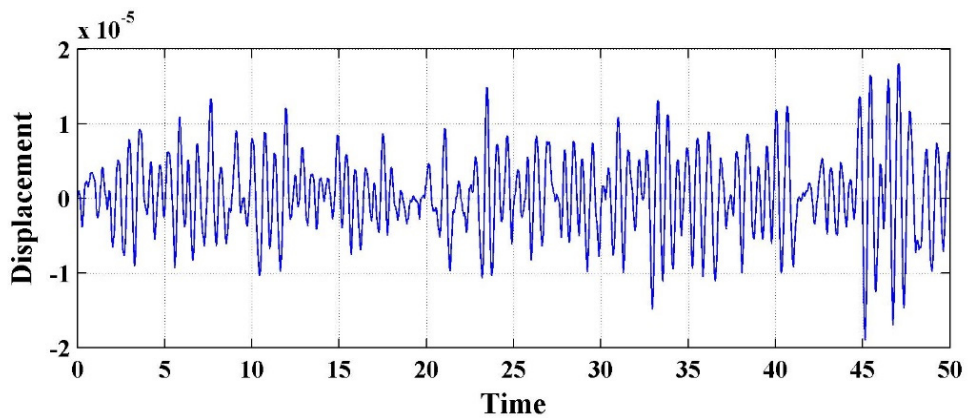

Fig. 9. The time history of the primary system with fractional-order derivative $\left(k_{2}=6000, c_{2}=100\right)$

\subsection{The comparison with the Voigt type DVA}

In order to verify the performance of the fractional-order DVA, the comparison between the fractional-order and the traditional integer-order Voigt type DVA is presented in this subsection.

The amplification factor of the integer-order Voigt type DVA is:

$$
A=\sqrt{\frac{\left(2 v \lambda \zeta_{2}\right)^{2}+\left(\nu^{2}-\lambda^{2}\right)^{2}}{\left(2 \lambda \nu \zeta_{2}\right)^{2}\left(1-\lambda^{2}-\mu \lambda^{2}\right)^{2}+\left[v^{2}-\left(1+v^{2}+\mu v^{2}\right) \lambda^{2}+\lambda^{4}\right]^{2}}}
$$

According to the reference [4], the optimal parameters of Voigt DVA for the $H_{\infty}$ optimization criterion is $\mu=0.1, v_{\text {opt }}=0.909, \zeta_{\text {2opt }}=0.185$.

The comparison of the fractional-order and the traditional integer-order Voigt type DVA is shown in Fig. 10. The parameters $k_{2}$ and $c_{2}$ are all selected as 0 in the fractional-order DVA so as to find out the effect of fractional-order derivative on the system clearly.

Similarly, one can choose the following parameters according to the existing literature [15] for the $H_{2}$ optimization criterion $\mu=0.1, v_{\text {opt }}=0.932, \zeta_{2 o p t}=0.153$.

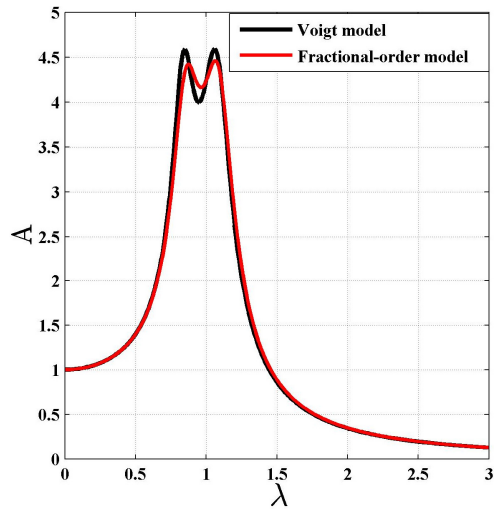

Fig. 10. The comparison of the fractional-order and integer-order Voigt type DVA for $H_{\infty}$ optimization criterion

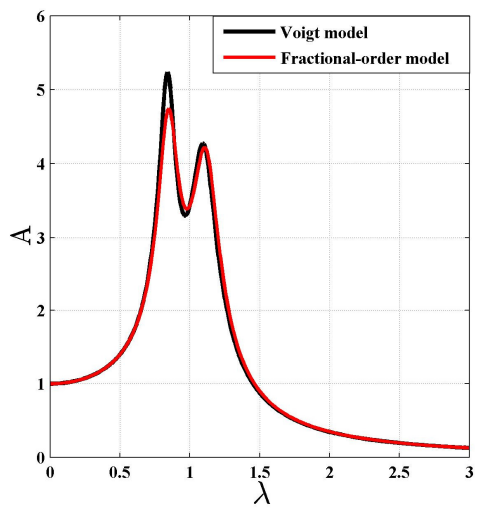

Fig. 11. The comparison of the fractional-order and integer-order Voigt type DVA for $H_{2}$ optimization criterion

To observe the effect of the fractional-order derivative, one can also select $k_{2}$ and $c_{2}$ to be zero. The comparison results are shown in Fig. 11. From Fig. 10 and Fig. 11, it could be found that in the two cases the amplitude-frequency curves of the fractional-order DVA agree well with those of the integer-order DVA, even when the linear stiffness and damping coefficients of the fractional-order DVA equal zero. That means the fractional-order derivative has a very good 
substitution for the stiffness and damping elements of DVA. The results also prove the correctness of the concept of equivalent stiffness and damping coefficients presented in references [29-31].

Yongjun Shen presented the whole structure of the article. Yongjun Shen and Haibo Peng wrote the draft manuscript. Shaofang Wen, Shaopu Yang, and Haijun Xing helped to improve the quality of the article in the preparation procedure.

\section{Conclusion}

The optimization of the fractional-order DVA is studied in this paper, and the optimal fractional order and fractional coefficient are obtained for the $H_{\infty}$ and $H_{2}$ optimization criteria. The comparisons of the approximately analytical solutions with the numerical solutions certify the satisfactory precision of the approximately analytical solutions. The comparisons with the integer-order DVA show that the fractional-order derivative can almost replace the linear stiffness and linear damping element, which may make the dynamical system much simpler. As the fractional-order derivative can accurately model many viscoelastic materials for vibration control engineering, the linear spring and damping elements may be replaced by one fractional-order element. These results may be very useful to design or revise vibration control device in engineering. Of course, it is important to study the replacement in engineering practice, which may be the next study object.

\section{Acknowledgements}

The authors are grateful to the support by National Natural Science Foundation of China (No. 11372198), the Cultivation Plan for Innovation Team and Leading Talent in Colleges and Universities of Hebei Province (LJRC018), the Program for Advanced Talent in the Universities of Hebei Province (GCC2014053), and the Program for Advanced Talent in Hebei Province (A201401001).

\section{References}

[1] Frahm H. Device for Damping Vibrations of Bodies. U.S. Patent, No. 989958, 30 October 1909.

[2] Ormondroyd J., Den Hartog J. P. The theory of the dynamic vibration absorber. ASME Journal of Applied Mechanics, Vol. 1928, Issue 50, 7, p. 9-22.

[3] Hahnkamm E. The damping of the foundation vibrations at varying excitation frequency. Ingenieur Archiv, Vol. 1932, 4, p. 192-201.

[4] Brock J. E. A note on the damped vibration absorber. ASME Journal of Applied Mechanics, Vol. 13, Issue 4, 1946, p. 284.

[5] Nishihara O., Asami T. Close-form solutions to the exact optimizations of dynamic vibration absorber (minimizations of the maximum amplitude magnification factors). ASME Journal of Vibration and Acoustics, Vol. 124, 2002, p. 576-582.

[6] Crandall S. H., Mark W. D. Random Vibration in Mechanical Systems. Academic Press, New York, 1963.

[7] Iwata Y. On the construction of the dynamic vibration absorbers. Japan Society of Mechanical Engineers, Vol. 820, Issue 8, 1982, p. 150-152.

[8] Asami T. Optimum design of dynamic absorbers for a system subjected to random excitation. JSME International Journal, Vol. 34, Issue 2, 1991, p. 218-226.

[9] Yamaguchi H. Damping of transient vibration by a dynamic absorber. Transactions of the Japan Society of Mechanical Engineers Series C, Vol. 54, Issue 499, 1988, p. 561-568.

[10] Ioi T., Ikeda K. On the dynamic vibration damped absorber of the vibration system. Bulletin of The Japan Society of Mechanical Engineers, Vol. 21, Issue 151, 1978, p. 64-71.

[11] Randall S. E., Halsted D. M., Taylor D. L. Optimum vibration absorbers for linear damped systems. ASME Journal of Mechanical Design, Vol. 103, 1981, p. 908-913.

[12] Thompson A. G. Optimum tuning and damping of a dynamic vibration absorber applied to a force excited and damped primary system. Journal of Sound and Vibration, Vol. 77, Issue 3, 1981, p. 403-415. 
[13] Warburton G. B. Optimal absorber parameters for various combinations of response and excitation parameters. Earthquake Engineering and Structural Dynamics, Vol. 10, 1982, p. 381-401.

[14] Nishihara O., Matsuhisa H. Design and tuning of vibration control devices via stability criterion. Japan Society Mechanical Engineering, Vol. 97, Issue 10, 1997, p. 165-168.

[15] Asami T., Nishihara O., Baz A. M. Analytical solutions to $\mathrm{H}_{\infty}$ and $\mathrm{H}_{2}$ optimization of dynamic vibration absorbers attached to damped linear systems. ASME Journal of Vibration and Acoustics, Vol. 124, 2002, p. 284-295.

[16] Asami T., Nishihara O. Closed-form exact solution to $\mathrm{H}_{\infty}$ optimization of dynamic vibration absorbers application to different transfer functions and damping systems. ASME Journal of Vibration and Acoustics, Vol. 125, Issue 3, 2003, p. 398-405.

[17] Oldham K. B., Spanier J. The Fractional Calculus Theory and Applications of Differentiation and Integration to Arbitrary Order. Academic Press, New York, 1974.

[18] Podlubny I. Fractional Differential Equations. Academic Press, London, 1999.

[19] Petras I. Fractional-Order Nonlinear System. Higher Education Press, Beijing, 2011.

[20] Mandelbrot B. B. Les Objets Fractals: Forme, Hasard et Dimension. Flammarion, Paris, 1975.

[21] Machado J. A. T., Galhano A. M. S. Fractional dynamics: a statistical perspective. ASME Journal of Computational and Nonlinear Dynamics, Vol. 3, Issue 2, 2008, p. 021201

[22] Li G., Zhu Z., Cheng C. Dynamical stability of viscoelastic column with fractional derivative constitutive relation. Applied Mathematics and Mechanics, Vol. 22, Issue 3, 2001, p. 294-303.

[23] Wang Z., Hu H. Stability of a linear oscillator with damping force of fractional order derivative. Science China Physics, Mechanics and Astronomy, Vol. 53, Issue 2, 2010, p. 345-352.

[24] Wang Z., Du M. Asymptotical behavior of the solution of a SDOF linear fractionally damped vibration system. Shock and Vibration, Vol. 18, 2011, p. 257-268.

[25] Rossikhin Y. A., Shitikova M. V. Application of fractional derivatives to the analysis of damped vibrations of viscoelastic single mass systems. Acta Mechanica, Vol. 120, 1997, p. 109-125.

[26] Cao J., Ma C., Xie H., Jiang Z. Nonlinear dynamics of Duffing system with fractional order damping. ASME Journal of Computational and Nonlinear Dynamics, Vol. 5, 2010, p. 041012

[27] Sheu L. J., Chen H. K., Chen J. H., Tam L. M. Chaotic dynamics of the fractionally damped Duffing equation. Chaos, Solitons and Fractals Vol. 32, 2007, p. 1459-1468.

[28] Wahi P., Chatterjee A. Averaging oscillations with small fractional damping and delayed terms. Nonlinear Dynamics, Vol. 38, 2004, p. 3-22.

[29] Shen Y. J., Yang S. P., Xing H. J. Dynamical analysis of linear single degree-of-freedom oscillator with fractional-order derivative. Acta Physica Sinica, Vol. 61, Issue 11, 2012, p. 110505.

[30] Shen Y. J., Yang S. P., Xing H. J., Ma H. X. Primary resonance of Duffing oscillator with two kinds of fractional-order derivatives. International Journal of Non-Linear Mechanics, Vol. 47, 2012, p. 975-983.

[31] Shen Y. J., Yang S. P., Xing H. J., Gao G. S. Primary resonance of Duffing oscillator with fractional-order derivative. Communications in Nonlinear Science and Numerical Simulation, Vol. 17, 2012, p. 3092-3100.

[32] Chen L., Hu F., Zhu W. Stochastic dynamics and fractional optimal control of quasi integrable Hamiltonian systems with fractional derivative damping. Fractional Calculus and Applied Analysis, Vol. 16, Issue 1, 2013, p. 189-225.

[33] Chen L. C., Zhu W. Q. Stochastic jump and bifurcation of Duffing oscillator with fractional derivative damping under combined harmonic and white noise excitations. International Journal of Non-linear Mechanics, Vol. 46, Issue 12, 2011, p. 1324-1329.

[34] Chen L. C., Li H. F., Li Z. S., Zhu W. Q. First passage failure of single-degree-of-freedom nonlinear oscillators with fractional derivative. Journal of Vibration and Control, Vol. 19, Issue 14, 2013, p. 2154-2163.

[35] Chen L. C., Wang W. H., Li Z. S., Zhu W. Q. Stationary response of Duffing oscillator with hardening stiffness and fractional derivative. International Journal of Non-Linear Mechanics, Vol. 48, Issue 1, 2013, p. 44-50.

[36] Achar N., Lorenzo C. F., Hartley T. T. Initialization and the Caputo Fractional Derivative. NASA John H. Glenn Research Center at Lewis Field Report, 2003.

[37] Ortigueira M. D., Coito F. J. Initial conditions: what are we talking about? 3rd IFAC Workshop on Fractional Differentiation and its Applications, Ankara, Turkey, 2008. 
[38] Sabatier J., Merveillaut M., Malti R., Oustaloup A. On a representation of fractional order systems: interests for the initial condition problem. 3rd IFAC Workshop on Fractional Differentiation and its Applications, Ankara, Turkey, 2008.

[39] Burd V. Method of Averaging for Differential Equations on an Infinite Interval: Theory and Applications. Taylor and Francis Group, 2007.

[40] Shen Y. J., Zhao Y. X., Yang S. P., Xing H. J. A revised averaging method and general forms of approximate solution for nonlinear oscillator with only polynomial-type displacement nonlinearity. Journal of Vibroengineering, Vol. 16, Issue 4, 2014, p. 1864-1876.

\section{Appendix}

In this appendix the detail derivation procedure for Eq. (13a) is presented and the other equations in Eq. (13) can be obtained as the similar way. According to the averaging method, one could obtain:

$\dot{a}_{12}=\lim _{T \rightarrow \infty} \frac{\mu K}{T \omega} \int_{0}^{T}\left\{D^{p}\left[a_{2} \cos \varphi_{2}\right] \sin \varphi_{1}\right\} d \varphi_{1}$.

Based on the definition of fractional-order derivative, one can translate Eq. (A1) into the following equation:

$\dot{a}_{12}=-\frac{\mu K a_{2}}{\Gamma(1-p)} \lim _{T \rightarrow \infty} \frac{1}{T} \int_{0}^{T}\left\{\left[\int_{0}^{t} \frac{\sin \left(\omega u+\theta_{2}\right)}{(t-u)^{p}} d u\right] \sin \left(\omega u+\theta_{1}\right)\right\} d t$.

Letting $s=t-u$ and $d s=-d u$, one could obtain $u=t-s$ and $\omega u=\omega t-\omega s$. Accordingly, Eq. (A2) becomes:

$$
\begin{aligned}
\dot{a}_{12} & =-\frac{\mu K a_{2}}{\Gamma(1-p)} \lim _{T \rightarrow \infty} \frac{1}{T} \int_{0}^{T}\left\{\left[\int_{0}^{t} \frac{\sin \left(\omega t-\omega s+\theta_{2}\right)}{s^{p}} d s\right] \sin \left(\omega t+\theta_{1}\right)\right\} d t \\
& =-\frac{\mu K a_{2}}{\Gamma(1-p)} \lim _{T \rightarrow \infty} \frac{1}{T} \int_{0}^{T}\left\{\left[\int_{0}^{t} \frac{\cos (\omega s)}{s^{p}} d s\right] \sin \left(\omega t+\theta_{2}\right) \sin \left(\omega t+\theta_{1}\right)\right\} d t \\
& +\frac{\mu K a_{2}}{\Gamma(1-p)} \lim _{T \rightarrow \infty} \frac{1}{T} \int_{0}^{T}\left\{\left[\int_{0}^{t} \frac{\sin (\omega s)}{s^{p}} d s\right] \cos \left(\omega t+\theta_{2}\right) \sin \left(\omega t+\theta_{1}\right)\right\} d t .
\end{aligned}
$$

Defining the first and second part of Eq. (A3) as $A_{1}$ and $A_{2}$ respectively, and integrating $A_{1}$ based on Leibniz integral rule, one can get:

$$
\begin{aligned}
A_{1} & =-\left.\frac{\mu K a_{2}}{\Gamma(1-p)} \lim _{T \rightarrow \infty}\left\{\frac{2 \omega t \cos \left(\theta_{1}-\theta_{2}\right)-\sin \left(2 \omega t+\theta_{1}+\theta_{2}\right)}{4 \omega T}\left[\int_{0}^{t} \frac{\cos (\omega s)}{s^{p}} d s\right]\right\}\right|_{0} ^{T} \\
& +\frac{\mu K a_{2}}{\Gamma(1-p)} \lim _{T \rightarrow \infty} \frac{1}{T} \int_{0}^{T} \frac{2 \omega t \cos \left(\theta_{1}-\theta_{2}\right)-\sin \left(2 \omega t+\theta_{1}+\theta_{2}\right)}{4 \omega} \cdot \frac{\cos (\omega t)}{t^{p}} d t
\end{aligned}
$$

Defining the first part and second part of $A_{1}$ as $A_{11}$ and $A_{12}$ respectively, one can find the simplified steady-state form of $A_{11}$ based on Eq. (12) and Leibniz integral rule:

$A_{11}=-\frac{\mu K a_{2} \omega^{p-1} \cos \left(\theta_{1}-\theta_{2}\right)}{2} \sin \left(\frac{p \pi}{2}\right)$.

Actually one can conclude that $A_{12}$ will become zero, based on the trigonometric identities and Eq. (12). The detail derivation is shown as: 


$$
\begin{aligned}
A_{12} & =\frac{\mu K a_{2}}{\Gamma(1-p)} \lim _{T \rightarrow \infty} \frac{1}{T} \int_{0}^{T} \frac{2 \omega t \cos \left(\theta_{1}-\theta_{2}\right)-\sin \left(2 \omega t+\theta_{1}+\theta_{2}\right)}{4 \omega} \cdot \frac{\cos (\omega t)}{t^{p}} d t \\
& =\frac{\mu K a_{2}}{4 \omega \Gamma(1-p)} \lim _{T \rightarrow \infty} \frac{1}{T} \int_{0}^{T}\left[2 \omega \cos \left(\theta_{1}-\theta_{2}\right) \frac{\cos (\omega t)}{t^{p-1}}-\sin \left(2 \omega t+\theta_{1}+\theta_{2}\right) \frac{\cos (\omega t)}{t^{p}}\right] d t \\
& =-\frac{\mu K a_{2}}{4 \omega \Gamma(1-p)} \lim _{T \rightarrow \infty} \frac{1}{T} \int_{0}^{T} \frac{\sin \left(2 \omega t+\theta_{1}+\theta_{2}\right) \cos (\omega t)}{t^{p}} d t=-\frac{\mu K a_{2}}{4 \omega \Gamma(1-p)} \\
& \cdot \lim _{T \rightarrow \infty} \frac{1}{T} \int_{0}^{T} \frac{\sin \left(\theta_{1}+\theta_{2}\right)[\cos (\omega t)+\cos (3 \omega t)]+\cos \left(\theta_{1}+\theta_{2}\right)[\sin (\omega t)+\sin (3 \omega t)]}{t^{p}} d t \\
& =0 .
\end{aligned}
$$

Accordingly, the first part of Eq. (A3) will be:

$$
A_{1}=-\frac{\mu K a_{2} \omega^{p-1} \cos \left(\theta_{1}-\theta_{2}\right)}{2} \sin \left(\frac{p \pi}{2}\right) \text {. }
$$

After similar procedure, one can get the second part of Eq. (A3):

$$
A_{2}=\frac{\mu K a_{2} \omega^{p-1} \sin \left(\theta_{1}-\theta_{2}\right)}{2} \cos \left(\frac{p \pi}{2}\right) \text {. }
$$

Thus, the result of $\dot{a}_{12}$ will be:

$$
\dot{a}_{12}=\frac{\mu K a_{2} \omega^{p-1}}{2}\left[\sin \left(\theta_{1}-\theta_{2}\right) \cos \left(\frac{p \pi}{2}\right)-\cos \left(\theta_{1}-\theta_{2}\right) \sin \left(\frac{p \pi}{2}\right)\right] .
$$

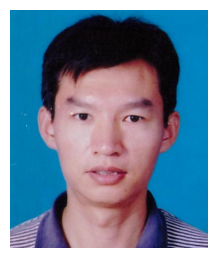

Yongjun Shen received the B.S. degree in Mechanical Engineering from Tianjin University, China, in 1995, his M.S. degree in Mechanical Engineering from Shijiazhuang Tiedao University, China, in 2002, and his Ph.D. degree in Vehicle Engineering from Beijing Jiaotong University, China, in 2006. He is a Professor in Department of Mechanical Engineering, Shijiazhuang Tiedao University. His research interests include nonlinear dynamics, vibration control, and fault diagnosis of mechanical system.

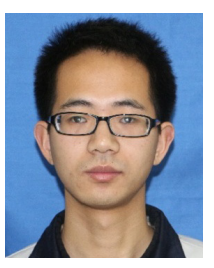

Haibo Peng received the B.S. and M.S. degree in Engineering Mechanics from Shijiazhuang Tiedao University, China, in 2012 and 2015. He is an Assistant Engineer in Great Wall Motor Company Limited, China. He is particularly interested in finite element simulation of vehicle safety and analytical solution for the complicated systems such as the fractional-order systems and nonlinear system.

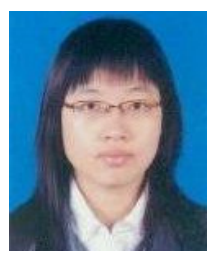

Shaofang Wen received the B.S. degree in Mechanical Engineering from Shijiazhuang Tiedao University, China, in 2002, and the M.S. degree in Mechanical Engineering from Shijiazhuang Tiedao University, China, in 2005. She is an Associate Professor in Transportation Institute, Shijiazhuang Tiedao University. Her research interests include the nonlinear dynamics and control in mechanical and transportation system. 


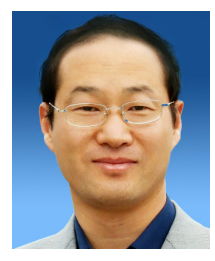

Shaopu Yang received his B.S. degree in Ocean Engineering from Tianjin University, China, in 1983, his M.S. and Ph.D. degrees in Dynamics and Control from Tianjin University, China, in 1985 and 1991 respectively. He is a Professor and President of Shijiazhuang Tiedao University. His research interests include nonlinear dynamics and vibration control of mechanical system, and vehicle-road coupled dynamics. His research achievements were awarded the National Science and Technology Award in 2003. His research was rewarded by the National Outstanding Young Scientist Fund of the National Natural Science Foundation of China. He is also the three-time winner of the Science and Technology Award of Hebei Province (2005, 2009, 2013).

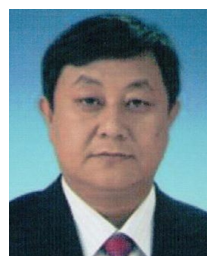

Haijun Xing received the B.S. degree in Mechanical Engineering from Dalian University of Technology, China, in 1990, his M.S. degree in Mechanical Engineering from Southwest Jiaotong University, China, in 1999, and his Ph.D. degree in Vehicle Engineering from Beijing Jiaotong University, China, in 2012. He is a Professor in Department of Mechanical Engineering, Shijiazhuang Tiedao University. His research interests include vibration control and optimization of mechanical system. 